\title{
ON NORMALLY FLAT EINSTEIN SUBMANIFOLDS
}

\author{
LEOPOLD VERSTRAELEN
}

\author{
K.U. Leuven \\ Department of Mathematics \\ Celestijnenlaan 200B \\ 3001 Leuven, Belgium
}

and

GEORGES ZAFINDRATAFA

Université de Valenciennes

Institut des Sciences et Techniques

B.P. 311, F-59304 Valencienes, Cedex, France

(Received December 21, 1990 and in revised form April 2, 1993)

\begin{abstract}
The purpose of this paper is to study the second fundamental form of some submanifolds $M^{n}$ in Euclidean spaces $\mathbb{E}^{m}$ which have flat normal connection. As such, Theorem 1 gives precise expressions for the (essentially 2) Weingarten maps of all 4-dimensional Einstein submanifolds in $\mathbb{E}^{6}$, which are specialized in Corollary 2 to the Ricci flat submanifolds. The main part of this paper deals with flat submanifolds. In 1919, E. Cartan proved that every flat submanifold of dimension $\leq 3$ in a Euclidean space is totally cylindrical. Moreover, he asserted without proof the existence of flat nontotally cylindrical submanifolds of dimension $>3$ in Euclidean spaces We will comment on this assertion, and in this respect will prove, in Theorem 3, that every flat submanifold $M^{n}$ with flat normal connection in $\mathbb{E}^{m}$ is totally cylindrical (for all possible dimensions $n$ and $m$ ).
\end{abstract}

KEY WORDS AND PHRASES. Submanifolds, normal connection, Ricci flat submanifolds. 1991 AMS SUBJECT CLASSIFICATION CODES. $\quad 53 \mathrm{C} 25$.

\section{INTRODUCTION.}

This paper deals first of all with the second fundamental form of an Einstein submanifold of codimension 2.

A Riemannian manifold is Einstein if its Ricci tensor field is proportional (with a constant coefficient of proportionality) to the Riemannian metric. We recall that every space of constant sectional curvature is Einstein

The converse statement is true also in 2 and 3 dimensions, as shown by J.A Schouten and D.J Struik in 1921

FACT A (see [8] or [5] or [1]). If a Riemannian manifold $M$ of dimension $n(n \leq 3)$ is Einstein, then it is a space of constant curvature.

T.Y. Thomas in 1936 and A. Fialkow in 1938 classified the Einstein hypersurfaces of the real space forms In particular, we have

FACT B (see [9] or [6] or [10] and [1]). Let $M^{n}$ be a hypersurface immersed in $\mathbb{E}^{n+1}$, where $n \geq 3$. If $M^{n}$ is Einstein, then:

(B.1) the Riemannian scalar curvature, say $s$, of $M$ is constant and non-negative,

(B.2) if $s=0$, then $M$ is locally Euclidean;

(B.3) if $s>0$, then every point of $M$ is umbilical and $M$ is locally a hypersphere $S^{m}$.

Theorem 1 of this paper determines all possible expressions of the second fundamental form of all Einstein 2-codimensional submanifolds with flat normal connection in $\mathbb{E}^{6}$, and in Corollary 2 we specify these expressions for all Ricci flat 2-codimensional submanifolds with flat normal connection in $\mathbb{E}^{6}$. The proofs of these two results use the flatness of the normal connection and are based on the following wellknown characterization of 4-dimensional Einstein spaces by I.M. Singer and T.A. Thorpe. 
FACT C (see [9] or [1]). Let $M$ be a Riemannian 4-manifold. Then $M$ is Einstein if and only if, for every $m \in M$, for any 2-plane $P$ at $m$, the sectional curvature of $P$ is equal to the sectional curvature of the 2-plane $P^{\perp}$ perpendicular to $P$ at $m$.

The method of the proof of Theorem 1 inspires us to establish in Theorem 3 a relation between flatness and cylindricity. The importance of this relation will be justified in Fact $D$.

\section{STATEMENTS OF THE MAIN RESULTS.}

THEOREM 1. Let $M$ be a 4-manifold isometrically immersed with flat normal connection in $\mathbb{E}^{6}$. Then $M$ is Einstein if and only if for each point $m \in M$ :

(1.1) either $M$ is cylindrical at $m$;

(1.2) or $M$ is umbilical (non-geodesic) with respect to a normal direction $N_{1}$ at $m$ and cylindrical in another normal direction $N_{2}$ perpendicular to $N_{1}$ at $m$;

(1.3) or with respect to a suitable orthonormal tangent frame of $M$ at $m$ and an orthonormal normal frame $\left\{N_{1}, N_{2}\right\}$ at $m$, the Weingarten operators $A_{N_{1}}, A_{N_{2}}$ admit respectively one among the following matricial representations:

$$
A_{N_{1}}=\left(\begin{array}{cccc}
a & 0 & 0 & 0 \\
0 & b & 0 & 0 \\
0 & 0 & 0 & 0 \\
0 & 0 & 0 & 0
\end{array}\right), \quad A_{N_{2}}=\left(\begin{array}{cccc}
0 & 0 & 0 & 0 \\
0 & 0 & 0 & 0 \\
0 & 0 & c & 0 \\
0 & 0 & 0 & d
\end{array}\right)
$$

where $a b=c d$;

$$
A_{N_{1}}=\left(\begin{array}{cccc}
a & 0 & 0 & 0 \\
0 & a & 0 & 0 \\
0 & 0 & -a & 0 \\
0 & 0 & 0 & -a
\end{array}\right),
$$

where $a$ is a non-zero real number, and $N_{2}$ is cylindrical;

$$
A_{N_{1}}=\left(\begin{array}{cccc}
a & 0 & 0 & 0 \\
0 & \frac{b}{a} & 0 & 0 \\
0 & 0 & \epsilon \frac{b}{a} & 0 \\
0 & 0 & 0 & 0
\end{array}\right), \quad A_{N_{2}}=\left(\begin{array}{cccc}
0 & 0 & 0 & 0 \\
0 & p & 0 & 0 \\
0 & 0 & q & 0 \\
0 & 0 & 0 & 0
\end{array}\right)
$$

where $\epsilon= \pm 1, a, b, p, q$ are real numbers such that $a b \neq 0$ and $p q=\epsilon\left(a^{2}-\frac{b^{2}}{a^{2}}\right)$;

$$
A_{N_{1}}=\left(\begin{array}{cccc}
a & 0 & 0 & 0 \\
0 & \frac{b}{a} & 0 & 0 \\
0 & 0 & \frac{c}{a} & 0 \\
0 & 0 & 0 & \frac{d}{a}
\end{array}\right), \quad A_{N_{2}}=\left(\begin{array}{cccc}
0 & 0 & 0 & 0 \\
0 & p & 0 & 0 \\
0 & 0 & q & 0 \\
0 & 0 & 0 & u
\end{array}\right)
$$

where $a, b, c, d, p, q, u$ are real numbers such that $a \neq 0$, and

$$
\begin{gathered}
p q=d-\frac{b c}{a^{2}}, \quad p u=c-\frac{b d}{a^{2}}, \\
q u=b-\frac{c d}{a^{2}}, \text { and }\left(b-\frac{c d}{a^{2}}\right) \cdot\left(c-\frac{b d}{a^{2}}\right) \cdot\left(d-\frac{b c}{a^{2}}\right)>0 .
\end{gathered}
$$

With respect to case 1.3.1 of Theorem 1, we give in particular the following

EXAMPLE AND REMARK 1. Let $M_{1}(c)$ and $M_{2}(c)$ be two surfaces of constant Gauss curvature $c$ in the Euclidean 3-space $\mathbb{E}^{3}$. Then

(1) the Riemannian product $M^{4}=M_{1}(c) \times M_{2}(c)$ canonically isometrically immersed in $\mathbb{E}^{6}$ is an Einstein 2-dimensional submanifold with flat normal connection. It is not a space of constant curvature and moreover it is not Ricci flat, unless $c=0$.

(2) In particular, for $c<0$, for instance $M_{1}(c)$ and $M_{2}(c)$ both being a pseudo-sphere in $\mathbb{E}^{3}$ of the same pseudo-radius $c$, the Riemannian product manifold $M^{4}$ is an Einstein submanifold with flat normal connection in $\mathbb{E}^{6}$ which has strictly negative scalar curvature. Thus, in contrast to the fact that for 1-codimensional Einstein submanifolds in Euclidean spaces the scalar curvature $s \in \mathbb{R}^{+}$, there exists 2codimensional Einstein submanifolds with any given real number as scalar curvature. 
COROLLARY 2. Let $M$ be a 4-dimensional manifold isometrically immersed with flat normal connection in $\mathbb{E}^{6}$.

Then $M$ is Ricci flat if and only if for each $m \in M$;

(2.1) either $M$ is flat (hence cylindrical) at $m$;

(2.2) or with respect to a suitable orthonormal tangent frame at $m$ and an orthonormal normal frame $\left\{N_{1}, N_{2}\right\}$ at $m$, the Weingarten operators $A_{N_{1}}, A_{N_{2}}$ admit respectively one of the following matricial representations:

$$
A_{N_{1}}=\left(\begin{array}{cccc}
a & 0 & 0 & 0 \\
0 & -\frac{a}{2} & 0 & 0 \\
0 & 0 & -\frac{a}{2} & 0 \\
0 & 0 & 0 & a
\end{array}\right), \quad A_{N_{2}}=\left(\begin{array}{cccc}
0 & 0 & 0 & 0 \\
0 & p & 0 & 0 \\
0 & 0 & q & 0 \\
0 & 0 & 0 & 0
\end{array}\right)
$$

where $p q=\frac{3}{4} a^{2}>0$.

$$
A_{N_{1}}=\left(\begin{array}{cccc}
a & 0 & 0 & 0 \\
0 & \frac{b}{a} & 0 & 0 \\
0 & 0 & \frac{c}{a} & 0 \\
0 & 0 & 0 & \frac{d}{a}
\end{array}\right), \quad A_{N_{2}}=\left(\begin{array}{cccc}
0 & 0 & 0 & 0 \\
0 & p & 0 & 0 \\
0 & 0 & q & 0 \\
0 & 0 & 0 & u
\end{array}\right)
$$

where $a \neq 0, p q=d-\frac{b c}{a^{2}}, p u=c-\frac{b d}{a^{2}}, q u=b-\frac{c d}{a^{2}}$ and $b+c+d=0,\left(d-\frac{b c}{a^{2}}\right) \cdot\left(c-\frac{b d}{a^{2}}\right) \cdot\left(b-\frac{c d}{a^{2}}\right)>0$.

THEOREM 3. Let $M^{n}$ be a n-dimensional manifold isometrically immersed with flat normal connection in $\mathbb{E}^{n+N}$.

Then $M^{n}$ is flat if and only if it is cylindrical.

3. DEFINITIONS [3]. $\mathbb{E}^{n+N}$

We consider a manifold $M$ isometrically immersed with codimension $N$ in the Euclidean space

3.1. Let $\xi$ be a normal vector field on $M$.

We shall say that $M$ is quasi-umbilical in the direction $\xi$ if the Weingarten tensor $A_{\xi}$ of $\xi$ admits an eigenvalue $\lambda_{\xi}$ with multiplicity $n-1$ or $n$.

In particular:

(i) if $\lambda_{\xi}=0$, we say that $M$ is cylindrical in the direction $\xi$;

(ii) if $\lambda_{\xi}$ has multiplicity $n$, we say that $M$ is umbilical in the normal direction $\xi$.

3.2. $M$ is (totally) cylindrical [resp. quasi-umbilical] if, locally around each point, there exists an orthonormal normal frame field composed with cylindrical [resp. quasi-umbilical] directions.

Now we prove our results.

\subsection{PROOF OF THE THEOREM 1.}

Let $M$ be a 4-manifold isometrically immersed in the Euclidean 6-space $\mathbb{E}^{6}$

Suppose that $M$ is Einstein. Then by Fact $\mathrm{C}$, for any $m \in M$ and any 2-plane $P$ in $T_{m} M$, its sectional curvature is the same as the sectional curvature of its orthogonal 2-plane $P^{\perp}$ in $T_{m} M$.

To exploit this statement, we suppose moreover that the normal connection of $M$ in $\mathbb{E}^{6}$ is flat Then, at each point $m \in M$, there exists an orthonormal tangent frame $\left\{e_{1}(m), \cdots, e_{4}(m)\right\}$ which diagonalizes simultaneously all Weingarten tensors of $M($ at $m)$. We denote by $c_{i j}(m)$ the sectional curvature of the 2-plane $\left\{e_{\imath}(m), e_{j}(m)\right\}$ for $1 \leq i<j \leq 4$. Then $M$ is Einsteinian if and only if for each $m \in M$.

$$
\left\{\begin{array}{l}
c_{12}(m)=c_{34}(m) \\
c_{13}(m)=c_{24}(m) \\
c_{14}(m)=c_{23}(m) .
\end{array}\right.
$$

Now we fix the point $m$ in $M$. Either $M$ is geodesic at $m$ : then the problem is solved. Or $M$ is non-geodesic at $m$; we can assume that $\sigma_{m}\left(e_{1}(m), e_{1}(m)\right) \neq 0$ where $\sigma_{m}$ is the second fundamental form at $m$. We can put $N_{1}=\frac{\sigma_{m}\left(e_{1}(m), e_{1}(m)\right)}{\left\|\sigma_{m}\left(e_{1}(m), e_{1}(m)\right)\right\|}$ and denote $N_{2}$ the unit normal vector perpendicular to $N_{1}$. By our choice of the tangent frame $\left\{e_{1}(m), \cdots, e_{4}(m)\right\}$, the Weingarten tensors $A_{N_{1}}, A_{N_{2}}$ relative to $N_{1}, N_{2}$ respectively can be represented by the matrices:

$$
A_{N_{1}}=\left(\begin{array}{cccc}
\lambda_{1} & 0 & 0 & 0 \\
0 & \lambda_{2} & 0 & 0 \\
0 & .0 & \lambda_{3} & 0 \\
0 & 0 & 0 & \lambda_{4}
\end{array}\right), \quad A_{N_{2}}=\left(\begin{array}{cccc}
0 & 0 & 0 & 0 \\
0 & \mu_{2} & 0 & 0 \\
0 & 0 & \mu_{3} & 0 \\
0 & 0 & 0 & \mu_{4}
\end{array}\right)
$$


Hence the previous system $\left({ }^{*}\right)$ is equivalent to the following one:

where $b=c_{12}=c_{34}, c=c_{13}=c_{24}, d=c_{14}=c_{23}$.

$$
\left\{\begin{array}{c}
\lambda_{1} \lambda_{2}=b(1) \\
\lambda_{1} \lambda_{3}=c(2) \\
\lambda_{1} \lambda_{4}=d(3) \\
\lambda_{3} \lambda_{4}+\mu_{3} \mu_{4}=b \\
\lambda_{2} \lambda_{4}+\mu_{2} \mu_{4}=c(4) \\
\lambda_{2} \lambda_{3}+\mu_{2} \mu_{3}=d(6)
\end{array}\right.
$$

To resolve this system of 6 equations with 7 unknowns, let us first compute $\lambda_{1}$ Using the equations (1), (2), (3) and the equality $b+c+d=\frac{1}{4} s$ (where $s$ is the constant scalar curvature of $M$ ) we find that $\lambda_{1}$ is a solution of the equation:

$$
x^{2}-4<H, N_{1}>x+\frac{1}{4} s=0
$$

where $x$ is unknown and $H$ is the mean curvature vector at $m$. Such an equation admits a solution $\lambda_{1}=a$ since:

$$
4<H, N_{1}>^{2}-\frac{1}{4} s=\left(\lambda_{1}+\lambda_{2}+\lambda_{3}+\lambda_{4}\right)^{2}-\lambda_{1}\left(\lambda_{2}+\lambda_{3}+\lambda_{4}\right) \geq 0 .
$$

To determine the unknowns $\lambda_{2}, \lambda_{3}, \lambda_{4}, \mu_{2}, \mu_{3}, \mu_{4}$, we discuss on the index of nonnullity $\pi(m)$ of $M$ at $m$, i.e., the rank of the Riemannian curvature operator $\mathcal{R}$ at $m$. Because of the system $\left(^{*}\right)$, $\pi(m) \in\{0,2,4,6\}$.

CASE 1: $\pi(m)=0$. Then $M$ is flat (hence Ricci flat) at $m$. By the system $\left({ }^{* *}\right), M$ is cylindrical at $m$.

CASE 2: $\pi(m)=2$. Then we obtain the situation (1.3.1).

CASE 3: $\pi(m)=4$. It is easy to check that this is impossible.

CASE 4: $\pi(m)=6$. From a simple discussion on the rank of $A_{N_{2}}$, we deduce either (1.2) or

\subsection{REMARK.} (1.3.2) or (1.3.3), or (1.3.4). This proves the Theorem 1

In accordance with each of the possibilities from Theorem 1 and Corollary 2, we can construct local parametrization of submanifolds of codimension 2 in $\mathbb{E}^{6}$ with flat normal connection which are, at a particular point, Einstein or in particular Ricci flat.

5. ON FLAT SUBMANIFOLDS.

5.1. A flat manifold is in particular Einstein. In 1919 [2], Elie Cartan studied the second fundamental form of flat submanifolds of a Euclidean space.

FACT D. ([2]). (D.1) Every $n$-dimensional flat submanifold;d of $\mathbb{E}^{n+N}$ with $n \leq 3$ is cylindrical. Moreover: (D.2) E. Cartan stated without proof, that the assertion (D.1) fails if $n \geq 4$.

With respect to (D.2), we consider the case of dimension $n=4$.

Assume $h: \mathbb{E}^{4} \times \mathbb{E}^{4} \rightarrow \mathbb{E}^{N}$ is a flat bilinear symmetric map and consider the dimension of the vector space $[\operatorname{Imh}]$ generated by the image of $h$. We may suppose without loss of generality that $N=\operatorname{dim}[\operatorname{Im} h]$. Since the dimension of the space of all symmetric bilinear forms on $\mathbb{E}^{4}$ is equal to 10 , we can restrict ourselves to $0 \leq N \leq 10$. Using techniques as for the proof of Fact (D.1), it is easy to demonstrate that, if $N \in\{7,8,9,10\}$, we can reduce $N$ so that $N \in\{0,1,2,3,4,5,6\}$. In the same paper where E. Cartan proved Fact (D.1), he showed also that for the case $N \in\{0,1,2,3,4\}$ the flatness implies the cylindricity. Consequently, the only unknown cases are: " $N=5$ " and " $N=6$ ". In 1986 [7], an example of a 4-submanifold in $\mathbb{E}^{10}$ which is, at a particular point, flat without being cylindrical is constructed. However, a full justification of Fact (D.2) is still lacking for the moment; in other words the method of resolution of the so-called Gauss equation of a flat submanifold in a Euclidean space is still unknown in dimension $n$ and in codimension $N$ with $N \geq n+1$, even for the case of dimension $n=4$. One first resolution for such a problem is given in Theorem 3 for the particular case of flat normal connection.

5.2. PROOF OF THEOREM 3. For this purpose, we apply the following Fact $\mathrm{E}$ and Lemma $\left(^{*}\right)$ which we state and prove below:

FACT E (see [7] and [2]). Let $\nu$ be a vector space. let $\omega$ be another vector space, endowed with a scalar product $\langle\cdot, \cdot\rangle$.

Suppose $\phi: \nu \times \nu \rightarrow \omega$ is a bilinear symmetric map, flat with respect to $\langle\cdot, \cdot\rangle$ (i.e., $\langle\phi(x, y), \phi(z, w)\rangle=\langle\phi(x, w), \phi(y, z)\rangle$ for any $x, y, z, w$ in $\nu$. Assume moreover that the orthogonal projection of $\phi$ on a subspace $W$ of $\omega$ is cylindrical. flat too.

Then the orthogonal projection of $\phi$ on the orthogonal supplementary subspace $W^{\perp}$ of $W$ in $\omega$ is 
LEMMA (*). Let $\sigma: \mathbb{E}^{n} \times \mathbb{E}^{n} \rightarrow \mathbb{E}^{N}$ be a flat bilinear symmetric map satisfying the following property (F): "There exists an "orthonormal frame $B=\left\{e_{1}, \cdots, e_{n}\right\}$ in $\mathbb{E}^{n}$ which diagonalizes simultaneously all projections $\langle\sigma, \xi\rangle$ of $\sigma$ in any direction $\xi \in \mathbb{E}^{N \text { ". }}$.

Then $\sigma$ is cylindrical.

PROOF. We shall prove this lemma by induction on $N$, and suppose $\sigma$ is not geodesic. The lemma is true for $N=1$.

Consider the case $N=2$. Let $\left\{\xi^{1}, \xi^{2}\right\}$ be an orthonormal frame in $\mathbb{E}^{2}$. The property $(\mathrm{F})$ implies that each component $\left\langle\sigma, \xi^{\alpha}\right\rangle$ can be represented in the frame $B$ by the matrix

$$
\left\langle\sigma, \xi^{\alpha}\right\rangle=\left(\begin{array}{cccc}
\lambda_{1}^{\alpha} & & & \\
& \lambda_{2}^{\alpha} & & 0 \\
0 & & \ddots & \\
& & & \lambda_{n}^{\alpha}
\end{array}\right) .
$$

The sectional curvature $c_{2}$ of each 2-plane generated by $\left\{e_{2}, e_{j}\right\}$ is given by

$$
c_{i j}=\sum_{\alpha=1}^{n} \lambda_{i}^{\alpha} \lambda_{j}^{\alpha} \text {. }
$$

We may suppose that $\sigma\left(e_{1}, e_{1}\right) \neq 0$ and $\xi^{2}$ is collinear to $\sigma\left(e_{1}, e_{1}\right)$. By this manner: $\lambda_{1}^{1}=0$ and $\lambda_{1}^{2} \neq 0$. Since $\sigma$ is flat, the $c_{\imath}$ are both null. This implies:

$$
\lambda_{i}^{2}=0 \text { for } 2 \leq i \leq n .
$$

Hence $\sigma$ is flat and $\left\langle\sigma, \xi^{2}\right\rangle$ is cylindrical. By Fact $\mathrm{E},\left\langle\sigma, \xi^{1}\right\rangle$ if flat too. Since $\left\langle\sigma, \xi^{1}\right\rangle$ is a (flat) bilinear symmetric form, it is well-known that it is cylindrical. Hence the lemma is proved for $N=2$.

Now suppose Lemma $\left(^{*}\right)$ is true for a certain integer $k$ and any dimension $N$ with $N \leq k$. Let us prove that it then is also true for $N=k+1$. By our hypothesis, $\sigma: \mathbb{E}^{n} \times \mathbb{E}^{n} \rightarrow \mathbb{E}^{k+1}$ if flat and enjoys the property (F). When we reason as for the case $N=2$, we easily find that $\left\langle\sigma, \xi^{k+1}\right\rangle$ is cylindrical. We apply Fact $E$ again and deduce that the projection $\sigma$ on the hypersurface $\mathbb{E}^{k}$ of $\mathbb{E}^{k+1}$ perpendicular to $\xi^{k+1}$ is flat too, $\sigma: \mathbb{E}^{n} \times \mathbb{E}^{n} \rightarrow \mathbb{E}^{k}$.

Our hypothesis of induction obviously asserts that $\sigma$ is cylindrical too. Hence $\sigma: \mathbb{E}^{n} \times \mathbb{E}^{n} \rightarrow \mathbb{E}^{k+1}$ is cylindrical. This completes the proof of Lemma $\left({ }^{*}\right)$.

6. OPEN PROBLEMS.

For the moment, the following questions related to this paper remain still without answer

PROBLEM 1. How to classify all Einstein 4-manifolds, and in particular all Ricci flat 4-manifolds? (see [1]).

PROBLEM 2. Resolve the Gauss equation of a flat submanifold $M^{4}$ of codimension 5 or 6 in the Euclidean space; i.e., find all bilinear symmetric map $\sigma: \mathbb{E}^{4} \times \mathbb{E}^{4} \rightarrow \mathbb{E}^{N}$ (for $N=5$ or 6 ) satisfying the equality: $\langle\sigma(x, y), \sigma(z, w)\rangle-\langle\sigma(x, z), \sigma(y, w)\rangle=0$ for any $x, y, z, w$ in $\mathbb{E}^{4}$ (consider only the case when the kernel $\operatorname{Ker} \sigma$ of $\sigma$ is trivial!).

ACKNOWLEDGEMENT. The second author would like to thank Professor Abdus Salam, the International Atomic Energy Agency and the UNESCO for hospitality at the International Centre for Theoretical Physics, Trieste, where most of the work on this article was done. The paper was finished when the second author was a Doctoral Research Fellow at the Katholieke Universiteit Leuven.

\section{REFERENCES}

1. BESSE, A.L., Einstein Manifolds, Ergebnisse der Mathematik and ihrer Grenzgebiete, SpringerVerlag, Berlin.

2. CARTAN, E., Sur les variétés de courbure constate d'un espace euclidien ou non euclidien, Bull. Soc. Math. 47 (1919), 125-160.

3. CHEN, B.Y., Geometry of Submanifolds, M. Dekkar, New York, 1973

4. FIALKOW, A., Hypersurfaces of a space of constant curvature, Ann. of Math. 39 (1938), 762-785

5. KOBAYASHI, S. \& NOMIZU, K., Foundations of Differential Geometry, Wiley, Interscience 1 , 1963.

6. KOBAYASHI, S. \& NOMIZU, K., Foundations of Differential Geometry, Wiley, Interscience 2, 1969.

7. MORVAN, J.M. \& ZAFINDRATAFA, G.K., Conformally flat submanifolds, Ann. Fac. Sci. Toulouse, VIII.3 (1986-87), 331-347.

8. SCHOUTEN, J.A. \& STRUIK D.J., On some properties of general manifolds relating to Einstein's theory of gravitation, Amer. J. Math. 43 (1921), 213-216.

9. SINGER, I.M. \& THORPE, J.A., The curvature of 4-dimensional Einstein spaces in global analysis, Papers in Honour of K. Kodaira, Princeton University Press, Princeton (1969), 355-365.

10. THOMAS, T.Y., On closed spaces of constant mean curvature, Amer. J. Math. 58 (1936), 702-704 


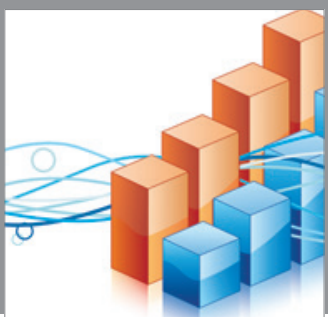

Advances in

Operations Research

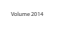

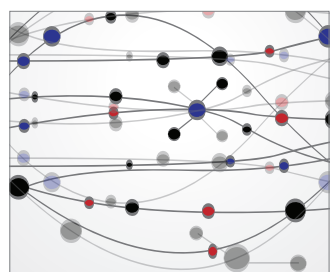

\section{The Scientific} World Journal
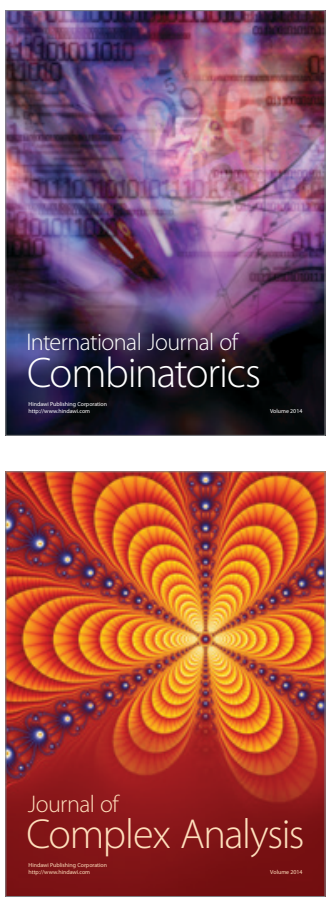

International Journal of

Mathematics and

Mathematical

Sciences
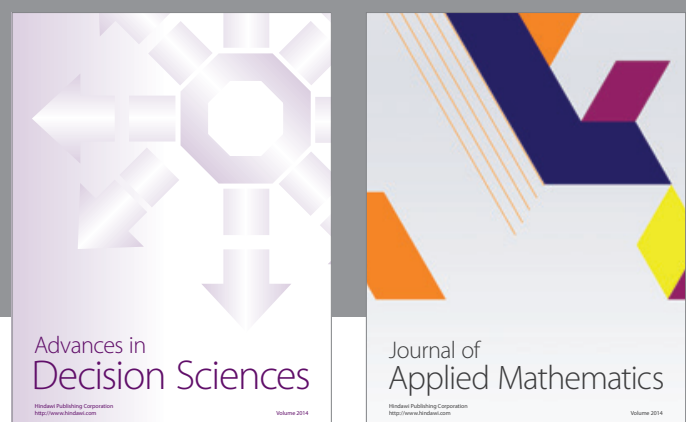

Journal of

Applied Mathematics
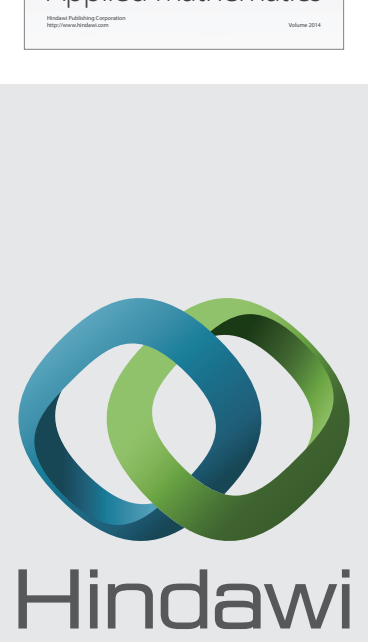

Submit your manuscripts at http://www.hindawi.com
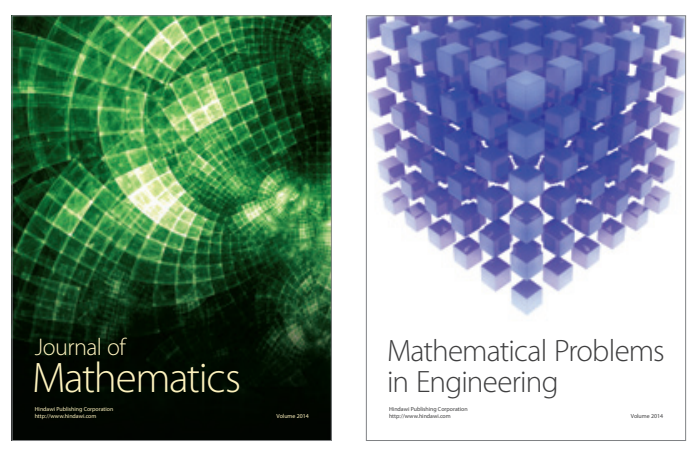

Mathematical Problems in Engineering
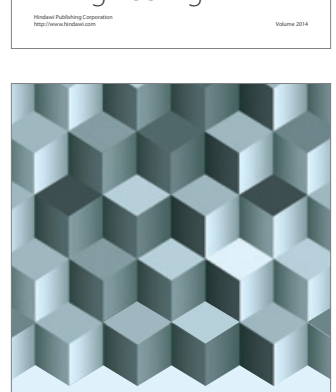

Journal of

Function Spaces
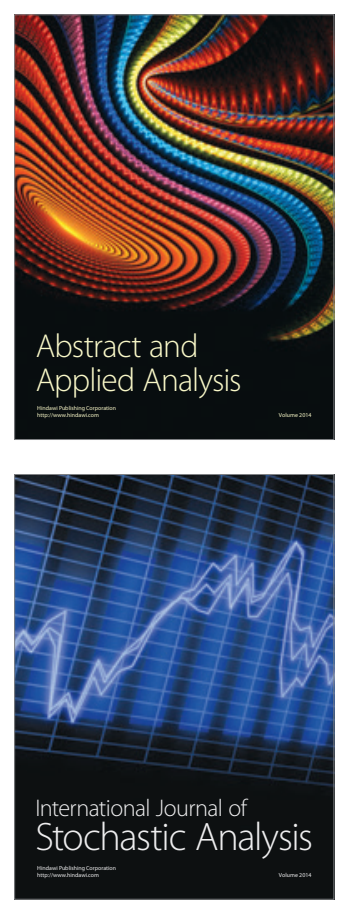

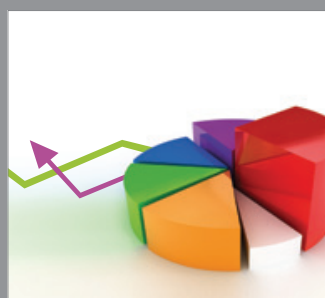

ournal of

Probability and Statistics

Promensencen
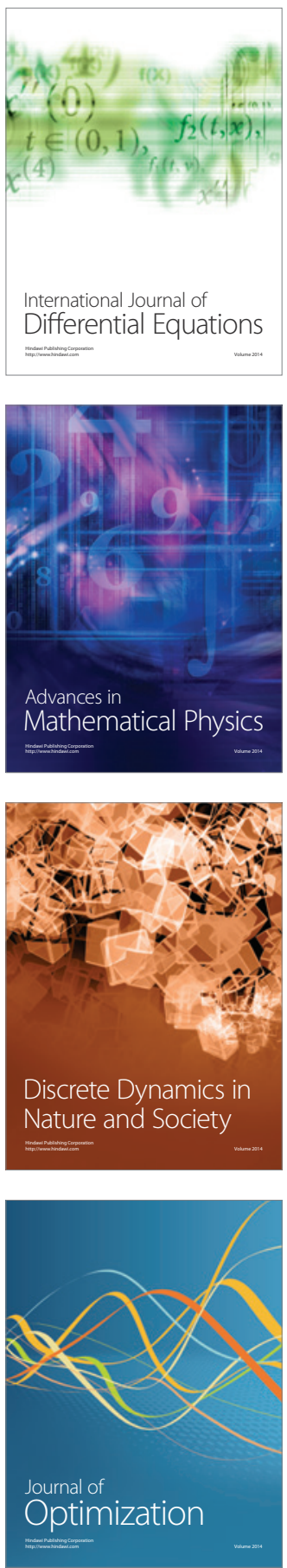